\title{
On the Development of Phi-Ro-Z Models with Physical Meaning using Monte Carlo Simulations
}

Raynald Gauvin

Department of Mining, Metals \& Materials Engineering, McGill University, 3610 University Street, Montréal, Québec, Canada, H3A 2B2

Quantitative x-ray microanalysis in the electron microprobe or in the scanning electron microscope is based on the following equation:

$$
\frac{\mathrm{C}_{\mathrm{i}}}{\mathrm{C}_{(\mathrm{i})}}=[\mathrm{ZAF}]_{\mathrm{i}} \frac{\mathrm{I}_{\mathrm{i}}}{\mathrm{I}_{(\mathrm{i})}}
$$

where $I_{i}$ is the net intensity of the characteristic line of element $i$ measured in the analyzed specimen of composition $\mathrm{C}_{\mathrm{i}}, \mathrm{I}_{(\mathrm{i})}$ is the net intensity of the same characteristic line of the same element measured on a standard of known composition $\mathrm{C}_{(\mathrm{i})}$ and $[\mathrm{ZAF}]_{\mathrm{i}}$ are the correction factors needed to solve equation (1). The modern approach is based on the use of $\varphi(\rho z)$ models to compute the $\mathrm{Z}$ and A factors. The first successful model was developed by Packwood \& Brown [1] and later improved by Bastin \& Heijligers [2]. This model is based on a Gaussian function to model the second part of the $\varphi(\rho z)$ curve, being justified by the fact that electrons reach a random walk behavior when they scatter into a solid. Figure [1] shows $\ln (\varphi(\rho z))$ versus $(\rho z)^{2}$ for the $\mathrm{M}_{5}$ shell of Au and for the $\mathrm{K}$ shell of $\mathrm{C}$ obtained from Monte Carlo simulations in bulk $\mathrm{Au}$ and $\mathrm{C}$ respectively at 20 $\mathrm{keV}$. A Gaussian behavior is justified for $\mathrm{Au}$ but not for $\mathrm{C}$ since a random walk behavior can not be obtained for that element [3]. Therefore, others models are needed for light elements despite the success of some models based on two parabolas [4] and on two exponential functions [5]. The success of these later models is due to extensive fitting of the parameters of these $\varphi(\rho z)$ models, especially for light elements. In order to obtain more reliable physical parameters for a $\varphi(\rho z)$ model that describes $x$-ray emission from a light matrix, a model with more physical meaning must be developed. In that context, we must start with an exact equation for the $\varphi(\rho z)$ curve for a pure element [6]:

$$
\varphi(\rho z)=n(\rho z) \frac{Q(E(\rho z))}{Q\left(E_{0}\right)}\langle\sec (\theta)(\rho z)\rangle
$$

Figure [2] shows a comparison of a Monte Carlo simulated $\varphi(\rho z)$ curve for $C \mathrm{~K}$ at 20 $\mathrm{keV}$ and with this computed with equation (2) with the 3 functions derived with the same simulation. Clearly, the agreement is excellent and a real physical $\varphi(\rho z)$ model should be based on equation (2).

\section{References}

1. R. H. Packwood and J. D. Brown (1981), X-Ray Spectr., 10, p. 138.

2. G. F. Bastin and H. J. M. Heijligers (1986), X-Ray Spectr., 15, p. 143.

3. R. Gauvin (1994), Scanning, 17, p. 348.

4. J. L. Pouchou and F. Pichoir (1987), Proc. $11^{\text {th }}$ ICXOM, p. 249.

5. J. L. Pouchou and F. Pichoir (1988), Microbeam Analysis, p. 315.

6. R. Gauvin (2005), paper in preparation. 


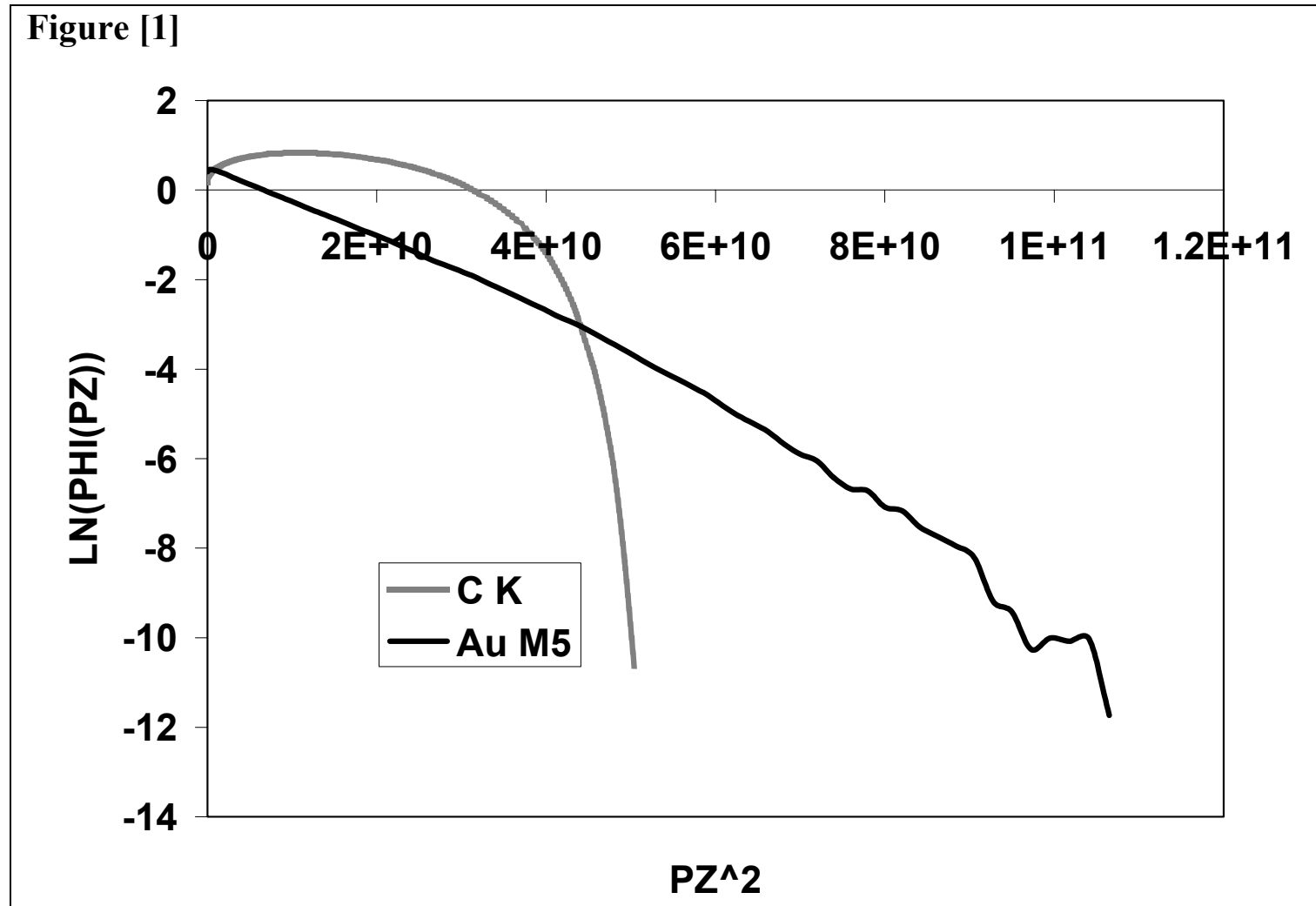

Figure [2]

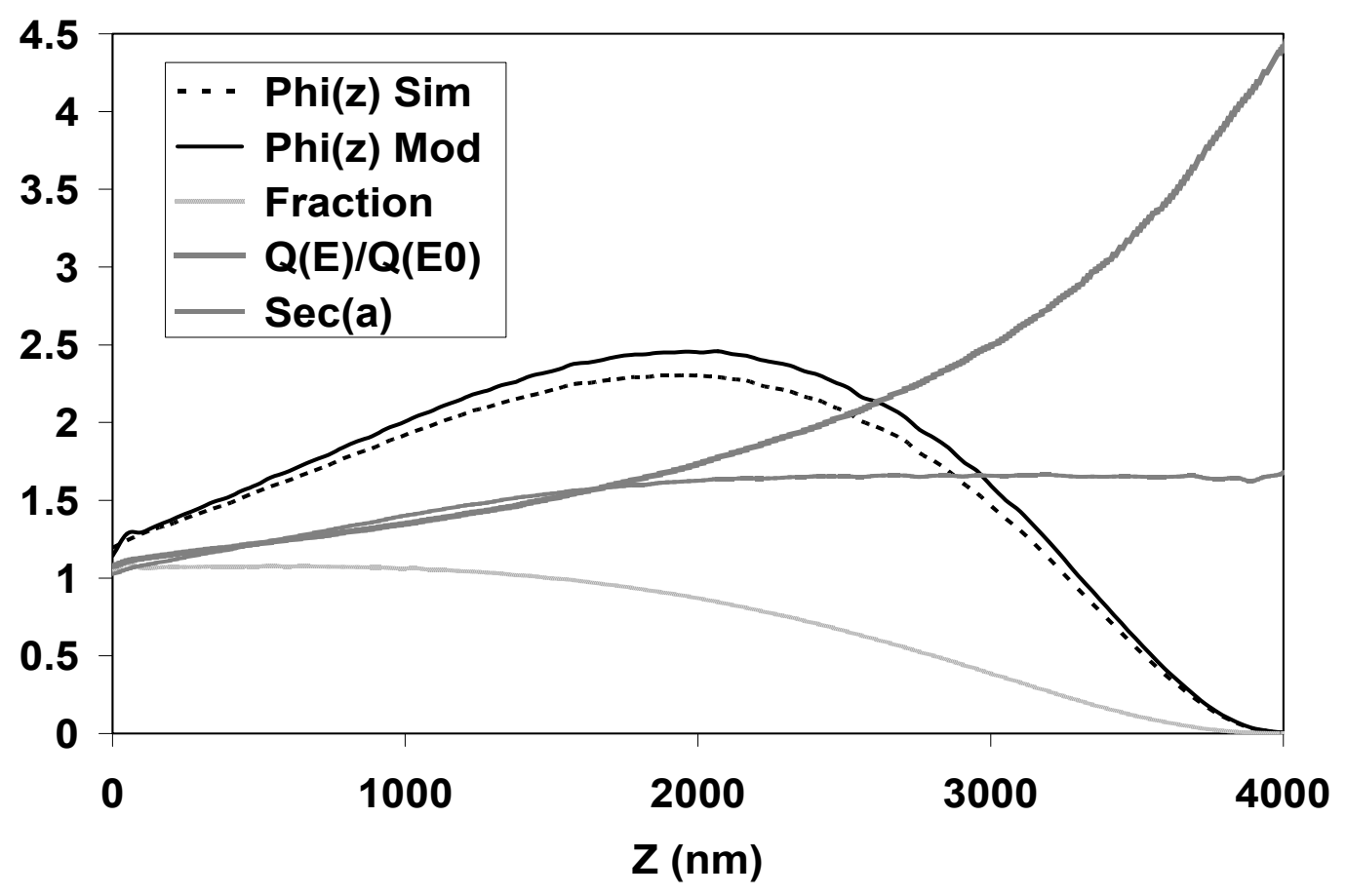

